\title{
Pregnancy in human IAPP transgenic mice recapitulates beta cell stress in type 2 diabetes
}

\author{
Tatyana Gurlo $^{1} \cdot$ Sarah Kim ${ }^{1} \cdot$ Alexandra E. Butler ${ }^{1} \cdot$ Chang Liu $^{1} \cdot$ Lina Pei $^{1} \cdot$ Madeline Rosenberger $^{1} \cdot$ Peter C. Butler $^{1}$
}

Received: 18 October 2018 / Accepted: 7 February 2019 / Published online: 9 March 2019

(C) Springer-Verlag GmbH Germany, part of Springer Nature 2019

\begin{abstract}
Aims/hypothesis Islet amyloid polypeptide (IAPP) misfolding and toxic oligomers contribute to beta cell loss and stress in type 2 diabetes. Pregnancy-related diabetes predicts subsequent risk for type 2 diabetes but little is known about the impact of pregnancy on beta cell mass, turnover and stress. Availability of human pancreas tissue in pregnancy is limited and most widely used mouse models of type 2 diabetes do not develop pregnancy-related diabetes, possibly because rodent IAPP is not prone to form toxic oligomers. We hypothesised that mice transgenic for human IAPP (hIAPP) are prone to pregnancy-related diabetes with beta cell responses reflective of those in type 2 diabetes.

Methods We evaluated the impact of a first and second pregnancy on glucose homeostasis, beta cell mass and turnover and markers of beta cell stress in hIAPP transgenic (hTG) mice.

Results Pregnancy induced both endoplasmic reticulum stress and oxidative stress and compromised autophagy in beta cells in hTG mice, which are characteristic of beta cells in type 2 diabetes. Beta cell stress persisted after pregnancy, resulting in subsequent diabetes before or during a second pregnancy.

Conclusions/interpretation High expression of hIAPP in response to pregnancy recapitulates mechanisms contributing to beta cell stress in type 2 diabetes. We hypothesise that, in individuals prone to type 2 diabetes, pregnancy-induced increased expression of IAPP inflicts beta cell damage that persists and is compounded by subsequent additive stress such as further pregnancy. The hTG mouse model is a novel model for pregnancy-related diabetes.
\end{abstract}

Keywords Beta cell mass · Gestational diabetes · Pregnancy $\cdot$ Type 2 diabetes

\begin{tabular}{ll}
\multicolumn{2}{l}{ Abbreviations } \\
CHOP & C/EBP homologous protein \\
ER & Endoplasmic reticulum \\
hIAPP & Human IAPP \\
hTG & hIAPP transgenic \\
IAPP & Islet amyloid polypeptide \\
p-H2AX & Phosphorylated H2A histone family member X
\end{tabular}

Electronic supplementary material The online version of this article (https://doi.org/10.1007/s00125-019-4843-z) contains peer-reviewed but unedited supplementary material, which is available to authorised users.

Peter C. Butler

pbutler@mednet.ucla.edu

1 Larry L. Hillblom Islet Research Center, David Geffen School of Medicine, University of California Los Angeles, 10833 Le Conte Avenue, 32-150 CHS, Los Angeles, CA 90095-7073, USA

\section{Introduction}

The third trimester of pregnancy is an adaptive state of insulin resistance that serves to preferentially direct nutrients to the rapidly growing fetus [1]. In health, maternal insulin secretion increases and glucose homeostasis is maintained [2]. In women who develop gestational diabetes, the adaptive increase in insulin secretion is inadequate [3-8]. This failure to increase insulin secretion sufficiently during pregnancy is predictive of subsequent risk of developing type 2 diabetes $[3,4,9,10]$. Moreover, multiparity increases the risk of subsequent diabetes [11-13], implying that pregnancy in those vulnerable to type 2 diabetes may have a lasting impact on maternal beta cells that persists beyond pregnancy. Collectively, these observations suggest that gestational diabetes represents an opportunity to investigate the mechanisms subserving beta cell failure in type 2 diabetes.

Two obstacles confront efforts to exploit that opportunity. First, there are limitations in accessing human pancreas from 


\section{Research in context}

\section{What is already known about this subject?}

- Gestational diabetes is a risk factor for subsequent type 2 diabetes

- Knowledge of pregnancy-related adaptive and maladaptive changes in beta cells in humans is limited by availability of human pancreas tissue in pregnancy

- Mouse models of diabetes previously used to investigate pregnancy-related diabetes are limited by failure to reproduce findings in beta cells in type 2 diabetes

\section{What is the key question?}

- Does pregnancy in the human islet amyloid polypeptide ( $h / A P P)$ transgenic mouse provide a more representative model of beta cell changes in human type 2 diabetes than previously used models?

\section{What are the new findings?}

- Pregnancy-induced expansion of beta cell mass in mice is comparable to that in humans if mice are aged beyond the juvenile period, during which there is a high capacity for beta cell replication

- In hIAPP transgenic mice, pregnancy-induced beta cell stress mimics the endoplasmic stress, oxidative damage and attenuated autophagy reported in beta cells in humans with type 2 diabetes

- This beta cell stress in hIAPP transgenic mice persists beyond pregnancy, resulting in subsequent diabetes that does not occur in non-mated controls, and/or diabetes in a second pregnancy

\section{How might this impact on clinical practice in the foreseeable future?}

- This study provides a model of spontaneous gestational diabetes that shares much in common with human gestational diabetes. It could be used for both screening/treatment strategies for gestational diabetes in humans as well as to assess the impact of gestational diabetes on fetal development

individuals during pregnancy. Second, rodents do not develop gestational diabetes comparable to humans [14]. A possible explanation is that beta cell failure in type 2 diabetes is characterised by protein misfolding toxicity mediated by islet amyloid polypeptide (IAPP). While human IAPP (hIAPP) is amyloidogenic and may form toxic oligomers in membranes, rodent IAPP is non-amyloidogenic [15]. In male rodents transgenic for $\mathrm{h} I A P P$, increased expression of hIAPP by high-fat feeding [16], pharmacological methods [17] or cross-breeding to hIAPP homozygosity leads to hIAPP protein misfolding [18], formation of intracellular toxic IAPP oligomers, beta cell failure and diabetes with an islet pathology comparable to that in type 2 diabetes [19]. In common with other rodent models of diabetes, female $\mathrm{h} I A P P$ transgenic mice are relatively protected from developing diabetes in an oestrogendependent manner [20]. However, we observed that homozygous hIAPP transgenic (hTG) female breeding mice were more prone to developing diabetes than non-mated mice, implying that this mouse model may provide a model for pregnancy-related diabetes.

Pregnancy, typically studied in mice aged $\sim 10-12$ weeks, has been used as a tool to investigate adaptive increases in beta cell mass [21]. Those data reveal that a $\sim$ two- to threefold increase in beta cell mass is achieved during murine pregnancy, mediated by a marked increase in beta cell replication.
However, the increase in beta cell mass in human pregnancy is only $\sim 1.4$ - to 2.0 -fold $[22,23]$. The capacity for beta cell replication declines in both humans (by age $\sim 2$ years) [24] and mice (by age $\sim 15$ weeks) $[25,26]$. We suspected that the difference in the adaptive expansion of beta cell mass between humans and young pregnant mice reflects the retained capacity for beta cell replication in mice at an age where pregnancy is possible compared with humans.

We first sought to establish whether pregnancy-related adaptive beta cell mass in mice more closely reflects that in humans if mice were studied at an age when the capacity for beta cell replication has declined comparably to the decline in humans. Second, we investigated the impact of pregnancy on beta cell mass and turnover in hTG mice, and to what extent this reproduced alterations in beta cells mass, turnover and stress reported in humans with type 2 diabetes.

\section{Methods}

\section{Mice}

The studies were reviewed and approved by the Office of Animal Research Oversight (OARO), UCLA, Los Angeles, CA, USA. The transgenic mice homozygous for human IAPP 
were originally from Pfizer (now available from Jackson Laboratory, Bar Harbor, ME, USA: IMSR cat. no. JAX:008232, RRID:IMSR_JAX:008232) and have been described elsewhere [18]. Wild-type FVB mice (IMSR cat. No. CRL:207, RRID:IMSR CRL:207) were originally purchased from Charles Rivers Laboratory (Wilmington, MA, USA). Both colonies were bred and maintained at UCLA on a 12 h day-night rhythm, with Harlan Teklad Rodent Diet 8604 and water ad libitum. The level of expression of IAPP in islets from female hTG mice assessed by western blot was $\sim 1$.6-fold higher than in wild-type mice (data not shown), similar to the level reported previously in the male hTG mouse type 2 diabetes model [27].

Blood glucose was measured weekly and on the day of euthanasia after overnight fasting from a tail-tip blood sample using a Freestyle blood glucose meter (Therasense, Alameda, CA, USA). A summary of body weight and blood glucose measurements is presented in electronic supplementary material (ESM) Fig. 1. Mice were euthanised using isoflurane. A mouse was considered diabetic if the fasting blood glucose level was higher than $7 \mathrm{mmol} / \mathrm{l}$. Eighty female FVB mice were used in this study: 30 were assigned to breed once and ten to breed twice; an additional 40 mice formed non-mated agematched control groups. In the hTG group, 82 mice were used in total: 44 were assigned to breed once and eight to breed twice; an additional 30 mice formed non-mated age-matched control groups. Mice characteristics on the day of tissue collection are presented in ESM Tables 1-4.

\section{Experimental design}

Age-dependence of adaptive response to pregnancy Blood glucose, beta cell mass and beta cell turnover were evaluated in a first pregnancy in young vs aged wild-type mice. The experiment plan is presented in Fig. 1a. Female wild-type mice were mated at age 8 weeks (young) or 26-28 weeks (aged). Twenty females from each age group were randomly assigned into pregnancy or non-mated control groups. Mice in the pregnancy group were mated and euthanised on day 15 post coitus; mice in the non-mated control group were euthanised at the same age.

Role of hIAPP toxicity in gestational diabetes The experiment plan is presented in Fig. 1b.

Protocol 1 explored the adaptive response to a first pregnancy. Blood glucose, beta cell mass and beta cell turnover were evaluated in a first pregnancy in hTG mice vs nontransgenic wild-type pregnant mice (the same young wildtype mice were used for the study of age-dependence of adaptive response to pregnancy described above). Mice were mated at 8 weeks of age and euthanised on day 15 post coitus. Corresponding non-mated hTG and wild-type control mice were evaluated in parallel. a

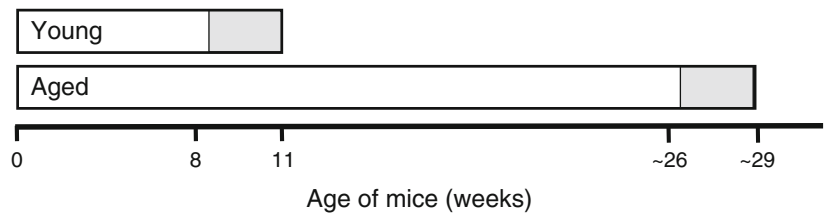

b
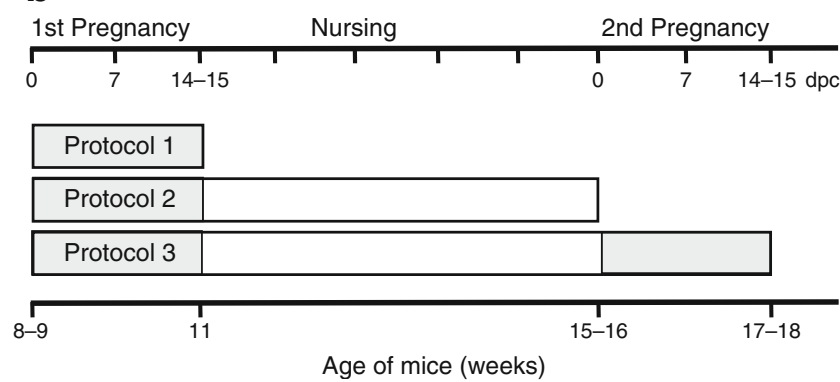

Fig. 1 Scheme of the experimental protocols related to the age-dependence of adaptive response to pregnancy (a) and the role of hIAPP in pregnancy-related diabetes (b). In Protocol 1, mice were mated at 8 weeks of age and pregnant mice were euthanised on the 15 th day post coitus. Non-mated mice of the same age ( $\sim 11$ weeks) were used as a control group. In Protocol 2, mice were mated at 8 weeks of age and were euthanised after delivery and nursing. Non-mated mice of the same age ( $\sim 15$ weeks) were used as a control group. In Protocol 3, mice were mated at 8 weeks of age. After delivery and nursing, mice that did not develop diabetes were mated again and euthanised on 15 th day post coitus. Nonmated mice of the same age ( $\sim 18$ weeks) were used as a control group. dpc, days post coitus

Protocol 2 explored the consequence of a first pregnancy on subsequent diabetes risk. Wild-type and hTG mice had blood glucose monitored throughout a first pregnancy (mated at 8 weeks of age) but were not euthanised until age 15 weeks to permit evaluation of beta cell mass and turnover post-pregnancy. Corresponding non-mated hTG and wild-type control mice were evaluated in parallel.

Protocol 3 explored the adaptive response to a second pregnancy. A group of hTG mice that did not develop diabetes after the first pregnancy and previously mated wild-type mice were mated again at 15 weeks of age. The mice were studied through a second pregnancy before euthanasia on day 15 post coitus for evaluation of beta cell mass and beta cell turnover. Corresponding non-mated hTG and wild-type control mice were evaluated in parallel.

\section{Pancreatic tissue processing and immunostaining}

The whole pancreas was rapidly resected, fat and nonpancreas tissue were trimmed and the pancreas was weighed. Pancreas was fixed in $4 \%$ paraformaldehyde overnight at $+4^{\circ} \mathrm{C}$ and embedded in paraffin for subsequent analysis as previously described [28]. Longitudinal sections of the pancreas (tail through head in the flat plane of the sections of pancreas) were then taken through the tissue in the plane of embedding so that a near-complete section of pancreas (head, 
body and tail) through its maximal width was obtained for each section. Sequential $4 \mu \mathrm{m}$ sections were stained as follows: (1) for insulin (peroxidase staining) and haematoxylin for beta cell mass (light microscopy); (2) TUNEL and insulin for cell death (immunofluorescence); (3) Ki67 and insulin for assessment of replication (immunofluorescence); (4) C/EBP homologous protein (CHOP) and insulin for assessment of endoplasmic reticulum (ER) stress (immunofluorescence); (5) p62 and insulin to assess the efficiency of protein degradation (immunofluorescence) and (6) phosphorylated H2A histone family member $\mathrm{X}(\mathrm{p}-\mathrm{H} 2 \mathrm{AX})$ and insulin for DNA damage (immunofluorescence). Additional sections were stained for insulin, IAPP and glucagon for assessment of islet morphology and IAPP expression (immunofluorescence).

Primary antibodies were: guinea pig anti-insulin 1:100 (Zymed, San Francisco, CA, USA; currently registered as Innovative Research cat. no. 18-0067, RRID:AB_86637); guinea pig anti-insulin 1:100 (Abcam, Cambridge, MA, USA; cat. no. ab7842, RRID:AB_306130); guinea pig anti-insulin 1:400 (Abcam cat. no. ab195956; Abcam; Cambridge, MA, USA; RRID not available); rabbit anti-IAPP 1:1000 for western blotting and 1:500 for immunofluorescence (Peninsula Laboratories cat. no. T-4157.0400, RRID:AB_518725); mouse anti-glucagon 1:1000 (Clone K79bB10, Sigma-Aldrich cat. no. G2654, RRID:AB_259852); rat anti-mouse Ki67 1:50 (TEC-3; Dako, Carpinteria, CA, USA; currently registered as Agilent cat. no. M7249, RRID:AB_2250503); rabbit anti-mouse CHOP 1:100 (GADD-153 [F168]; lot no. I0909; Santa Cruz Biotechnology cat. no. sc-575, RRID:AB_631365); guinea pig anti-p62 1:400 (Progen cat. no. GP62-C, RRID:AB_2687531); rabbit anti-PH2A.X Ser139 1:400 (clone 20E3; Cell Signaling Technology cat. no. 9718, RRID:AB_2118009). Secondary antibodies were $\mathrm{F}\left(\mathrm{ab}^{\prime}\right)_{2}$ conjugates with Alexa 647, Cy3 or FITC obtained from Jackson ImmunoResearch (West Grove, PA, USA), and used at a dilution of 1:200. For TUNEL staining, an in situ cell death detection kit (no. 12156792 910; Roche Diagnostics Corporation, Indianapolis, IN, USA) was used. For immunofluorescence, slides were mounted with Vectashield with DAPI (Vector Laboratories cat. no. H-1200, RRID:AB_2336790). For insulin peroxidase staining, the ABC-VECTASTAIN Standard Kit (Vector Laboratories cat. no. PK-4000, RRID:AB_2336818) and DAB Peroxidase Substrate Kit (Vector Laboratories cat. no. SK-4100, RRID:AB_2336382) from Vector Laboratories (Burlingame, CA, USA) was used; slides were mounted with Permount (SP15-100; Fisher, Hampton, NH, USA).

\section{Morphometric analysis}

Beta cell mass was calculated as beta cell fractional area multiplied by pancreas weight. To determine the pancreatic fractional beta cell area, the entire pancreatic section was imaged at $\times 40$ magnification ( $\times 4$ objective) using Olympus IX70 microscope
(Olympus America, Melville, NY, USA). The ratio of the beta cell area/exocrine area was digitally quantified as described [28] using Image Pro Plus software (Image Pro Plus version 5.1.0.20; Media Cybernetics, Silver Springs, MD, USA). The same approach was used for the assessment of alpha cell mass.

The mean cross-sectional area of beta cells was determined using sections stained for insulin (fluorescence). All cell clusters and single beta cells on the entire section were imaged using the Leica DM6000 microscope with $\times 20$ objective (Leica Microsystems, Buffalo Grove, IL, USA). The area stained for insulin in each cluster was measured using Image Pro Plus and the number of beta cells was counted. The area of single beta cells was calculated by dividing insulin positive area by number of beta cells within this area; $30-100$ clusters per section were analysed.

The frequencies of beta cell replication and death was determined using sections double-stained for Ki67 and insulin, and TUNEL and insulin, respectively. Sections were viewed by Leica DM6000 microscope (Leica Microsystems, Buffalo Grove, IL, USA) with $\times 20$ objective and all beta cells on the entire section were counted (1000-4500 cells per section for non-diabetic mice). In diabetic mice, because the number of beta cells per section was sometimes low, we analysed at least three nonadjacent sections to accumulate sufficient beta cell numbers for evaluation (a minimum of 1000 beta cells per mouse). The frequencies of beta cell replication and death was expressed as percentage of Ki67-positive beta cells and percentage of TUNEL-positive beta cells, respectively. The defect in the autophagy-lysosomal pathway of protein degradation was assessed by the frequency of beta cells containing p62-positive inclusions. The extent of ER stress and DNA damage were evaluated by the percentage of CHOP-positive or p-H2AX-positive beta cells counted on the sections co-stained for CHOP and insulin, and $\mathrm{p}-\mathrm{H} 2 \mathrm{AX}$ and insulin, respectively.

\section{Statistical analysis}

Data are presented as mean \pm SEM. Statistical calculations were carried out by one-way ANOVA followed by Fisher's least-squares difference post hoc test for multiple group comparison using Statistica 8.0 (Statsoft, Tulsa, OK, USA) or by two-tailed unpaired Student's $t$ test to compare two groups (in Fig. 2 only as indicated in the legend). A value of $p<0.05$ was considered statistically significant.

\section{Results}

\section{The adaptive increase in beta cell mass during pregnancy is attenuated with age}

To investigate the impact of age on adaptation of beta cell mass during pregnancy, we evaluated the increment in beta 
Fig. 2 Fasting blood glucose (a) and beta cell mass (b), crosssectional area (c), proliferation (d) and death (e) in young and aged pregnant and age-matched nonmated wild-type mice. White circles, young mice; black circles, aged mice. NM, non-mated mice; $\mathrm{P}$, pregnant mice. Data are the mean \pm SEM, $n=10$ for each group; $* p<0.05$ and $* * * p<0.001$, one-way ANOVA followed by Fisher's least-squares difference post hoc test; in (a) * $p<0.05$ was calculated using two-tailed unpaired Student's $t$ test
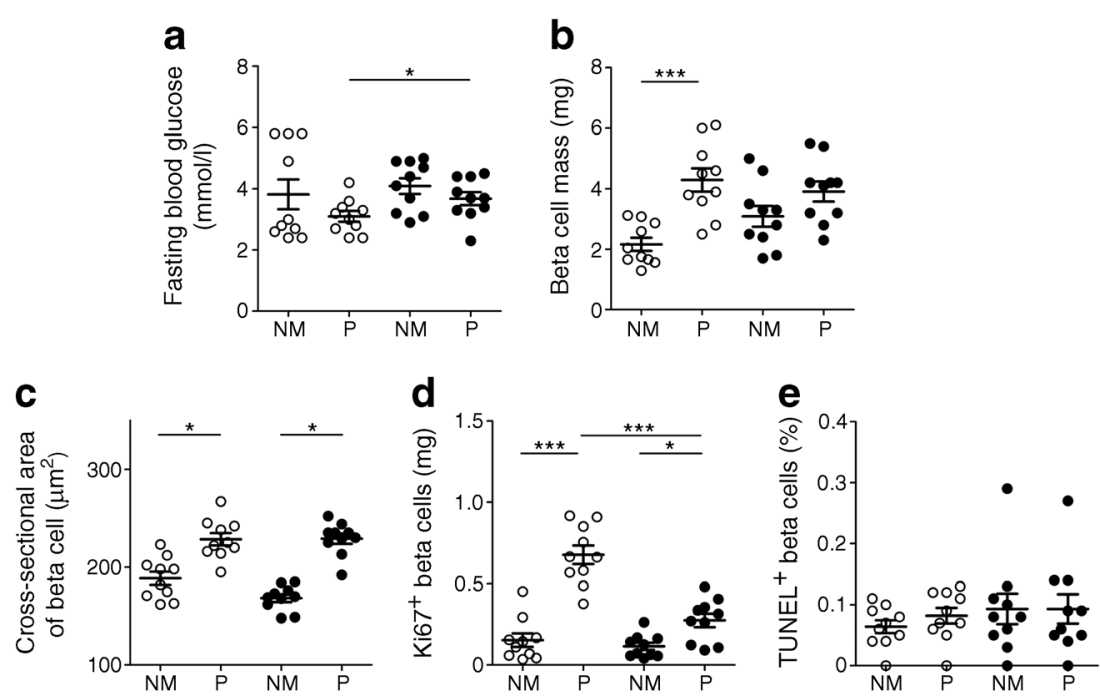

cell mass in young pregnant (aged 11 weeks old) vs aged pregnant (aged 28-30 weeks) mice in comparison with agematched non-mated littermates. Body weight and pancreas weight increased comparably with pregnancy in young and aged mice (ESM Table 1). Blood glucose did not differ between young non-mated and aged non-mated mice but levels were higher in aged pregnant vs young pregnant mice $(3.7 \pm$ 0.2 vs $3.1 \pm 0.2 \mathrm{mmol} / 1, p<0.05$; Fig. $2 \mathrm{a})$. Beta cell mass increased approximately twofold in young pregnant mice compared with young non-mated mice $(4.3 \pm 0.4$ vs $2.2 \pm$ $0.2 \mathrm{mg}, p<0.001$ ) but there was only a $\sim 1.3$-fold increase in aged pregnant mice vs aged non-mated mice $(3.9 \pm 0.3$ vs 3.1 $\pm 0.3 \mathrm{mg}, p<0.1$ ) (Fig. 2b). The increased beta cell mass seen in young pregnant mice, compared with young non-mated mice, was attributable to a $\sim 1.2$-fold increase in beta cell size (Fig. 2c) and a fourfold increase in beta cell replication $(0.68$ $\pm 0.06 \%$ vs $0.17 \pm 0.04 \%, p<0.001$; Fig. $2 d)$. The smaller increase in beta cell mass in pregnant aged mice vs nonmated aged mice was due to a smaller increment in beta cell replication $(0.27 \pm 0.04 \%$ vs $0.11 \pm 0.02 \%, p<0.05$; Fig. $2 d)$ since the increase in beta cell size was comparable in young $(\sim 1.2$-fold) and aged ( 1.4-fold) mice. Beta cell death was rarely detected and was not altered in pregnancy irrespective of age (Fig. 2e). Islet size distribution was similar in young non-mated and pregnant mice (ESM Fig. 2). In aged pregnant mice, small islets were proportionately more frequent than large islets in comparison with age-matched control mice, comparable to human pregnancy [22].

In conclusion, beta cell mass increases in response to pregnancy in young mice, through increased beta cell replication. Pregnancy-related increase in beta cell mass occurs to a lesser extent in aged mice in which the capacity to increase beta cell replication is attenuated. As such, the changes in beta cell mass in aged mice more closely mirror those observed in human pregnancy.

\section{Pregnancy increases hIAPP-induced beta cell toxicity, contributing to pregnancy-related diabetes}

Pregnancy is a risk factor for developing type 2 diabetes [4], a disease characterised by beta cell stress induced by hIAPP misfolding [28-30]. We sought to establish the potential contribution of hIAPP misfolding in beta cells in pregnancyinduced diabetes by comparing hTG mice (transgenically expressing $\mathrm{h} I A P P$ in beta cells) with wild-type mice (expressing soluble rodent IAPP).

First pregnancy (Protocol 1) During their first pregnancy, hTG mice were modestly hyperglycaemic compared with wild-type mice $(4.0 \pm 0.1$ vs $3.1 \pm 0.2 \mathrm{mmol} / 1, p<0.05$; Fig. 3a, Protocol 1). The increased fasting glucose in hTG mice during their first pregnancy, when compared with wild-type mice, was accompanied by a modest deficit in beta cell mass (Fig. 3b, Protocol 1) by virtue of the magnitude of the pregnancy-induced increase in beta cell mass (compared with non-mated mice) being smaller (1.3fold vs twofold) in the mice expressing hIAPP $(3.3 \pm 0.3$ vs $2.3 \pm 0.3 \mathrm{mg}, p<0.05)$ than in the wild-type mice $(4.3$ \pm 0.4 vs $2.2 \pm 0.2 \mathrm{mg}, p<0.001)$.

Beta cell mass was maintained in non-mated hTG mice by increased beta cell replication, which offset an increased frequency of beta cell death (Fig. 3c,d, Protocol 1). As TUNEL detects cell death by several mechanisms, including apoptosis, we used detection of cleaved cytokeratin 18 (CK18), a known substrate of caspase- 3 , to validate the finding that beta cell apoptosis was increased in hTG mice (ESM Fig. 3).

In contrast to the wild-type mice, hTG mice showed no further increment in beta cell replication during their first pregnancy, presumably accounting for the modest deficit in beta cell mass. Alpha cell mass was comparable between groups (ESM Fig. 4). The morphology of islets is shown in Fig. 4, Protocol 1. 
Fig. 3 Fasting blood glucose (a) and beta cell mass (b),

proliferation (c) and death (d) in wild-type and hTG mice during the first pregnancy (Protocol 1), after the first pregnancy (Protocol 2 ) and, for mice that did not develop diabetes during protocols 1 and 2, during the second pregnancy (Protocol 3). White circles, wild-type mice; black circles, hTG mice. NM, agematched non-mated mice; P1, first pregnancy; P2, second pregnancy. Data are the mean \pm SEM; $* p<0.05, * * p<0.01$ and $* * * p<0.001$, one-way ANOVA followed by Fisher's least-squares difference post hoc test. Protocol $1, n=10$ in all groups; Protocol 2, $n=8$ in wild-type NM group, $n=9$ in wild-type post-P1 group; $n=10$ in both groups of hTG mice in (a) and (b), $n=6$ in all groups in (c) and (d); Protocol 3, $n=7$ in hTGP2 group in (a) and (b), $n=10$ for all other groups
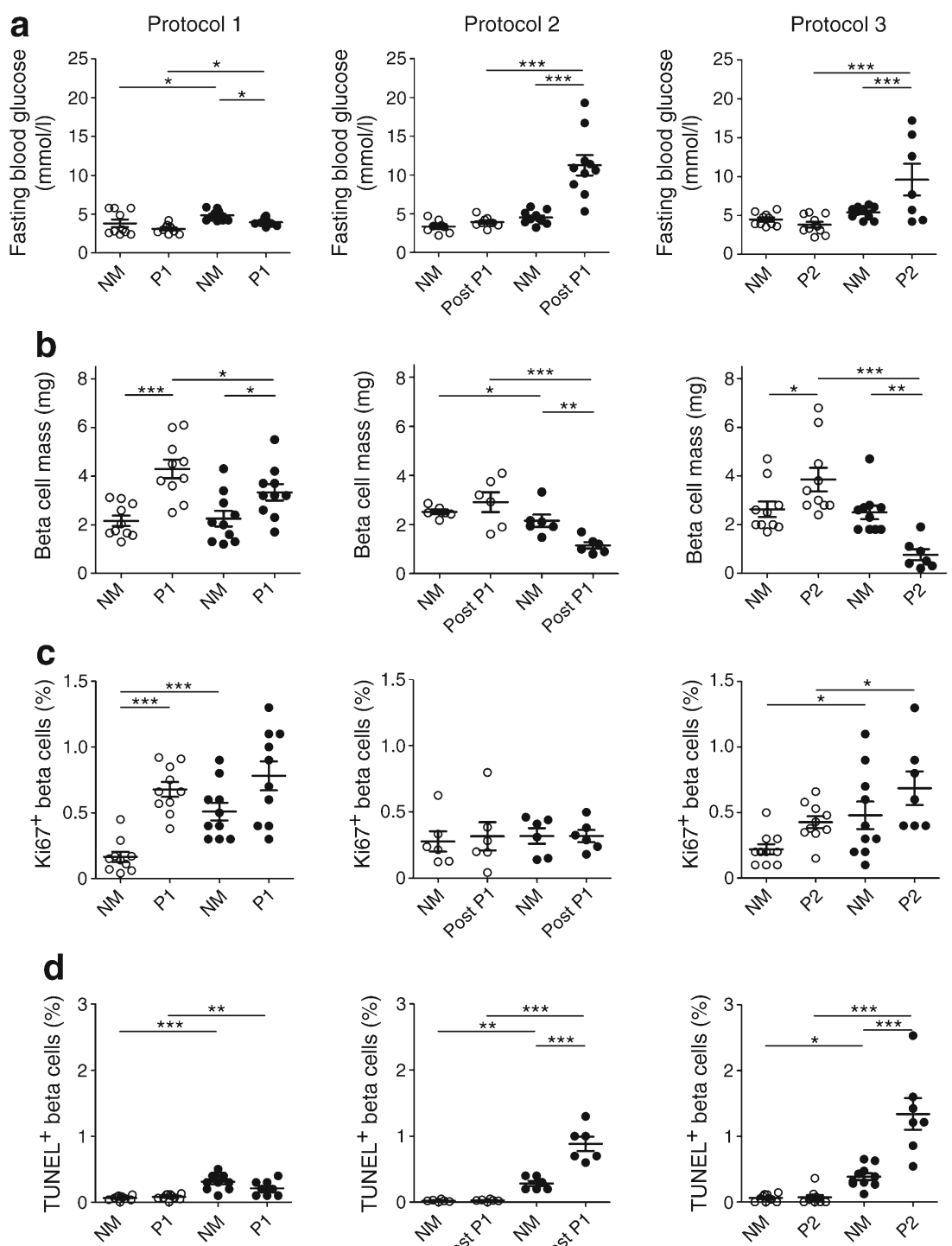

In conclusion, young mice transgenic for $\mathrm{h} I A P P$ have a modestly increased fasting blood glucose concentration and a $20 \%$ deficit in beta cell mass during a first pregnancy compared with pregnant wild-type mice, which do not express oligomeric-prone hIAPP. We next evaluated the impact of a first pregnancy on the subsequent risk for diabetes in hTG mice.

Prior pregnancy increases the frequency of postpartum diabetes in hTG mice (Protocol 2) Despite only modest fasting hyperglycaemia during a first pregnancy, $\sim 75 \%$ of the previously pregnant hTG mice developed diabetes postpartum by 15 weeks of age compared with none of the age-matched non-mated hTG mice $(25 / 34$ vs $0 / 10)$. To investigate the impact of a prior pregnancy on vulnerability to subsequent diabetes development, we quantified beta cell mass and turnover in the previously pregnant vs non-mated hTG mice and wild-type control mice. Previously pregnant wild-type mice had a beta cell mass comparable to that of the non-mated control mice (Fig. 3b, Protocol 2). In contrast, beta cell mass was decreased by $\sim 50 \%$ in hTG mice after the first pregnancy compared with non-mated hTG mice $(1.1 \pm 0.1$ vs $2.0 \pm 0.2 \mathrm{mg}, p<0.01)$. Alpha cell mass was comparable between groups (ESM Fig. 4). The morphology of islets is shown in Fig. 4, Protocol 2.

The mechanism underlying the defect in beta cell mass in the hTG mice was increased beta cell death $(0.88 \pm 0.11 \%$ in hTG mice post-first pregnancy vs 0.27 $\pm 0.04 \%$ in non-mated $\mathrm{hTG}$ mice and $0.03 \pm 0.01 \%$ in wild-type mice post-first pregnancy, $p<0.001$; Fig. 3d, Protocol 2).

The implication of these findings is that a period of transient misfolded hIAPP beta cell stress can lead to subsequent diabetes due to the residual impact of the prior beta cell injury. Since multiparity has been linked to an increased risk of type 2 diabetes [11-13], we investigated the impact of a second pregnancy on the hTG mice that remained non-diabetic after the first pregnancy. 
Fig. 4 Representative images of islets from wild-type and hTG mice from all protocols. Pancreatic sections were stained for IAPP (Cy3, red), insulin (Ins; FITC, green) and glucagon (Gluc; Alexa 647, white); nuclei were stained blue with DAPI. NM, agematched non-mated mice; P1, first pregnancy; $\mathrm{P} 2$, second pregnancy. The exposure time during IAPP imaging in hTG groups was decreased to avoid bleaching. Scale bars, $50 \mu \mathrm{m}$

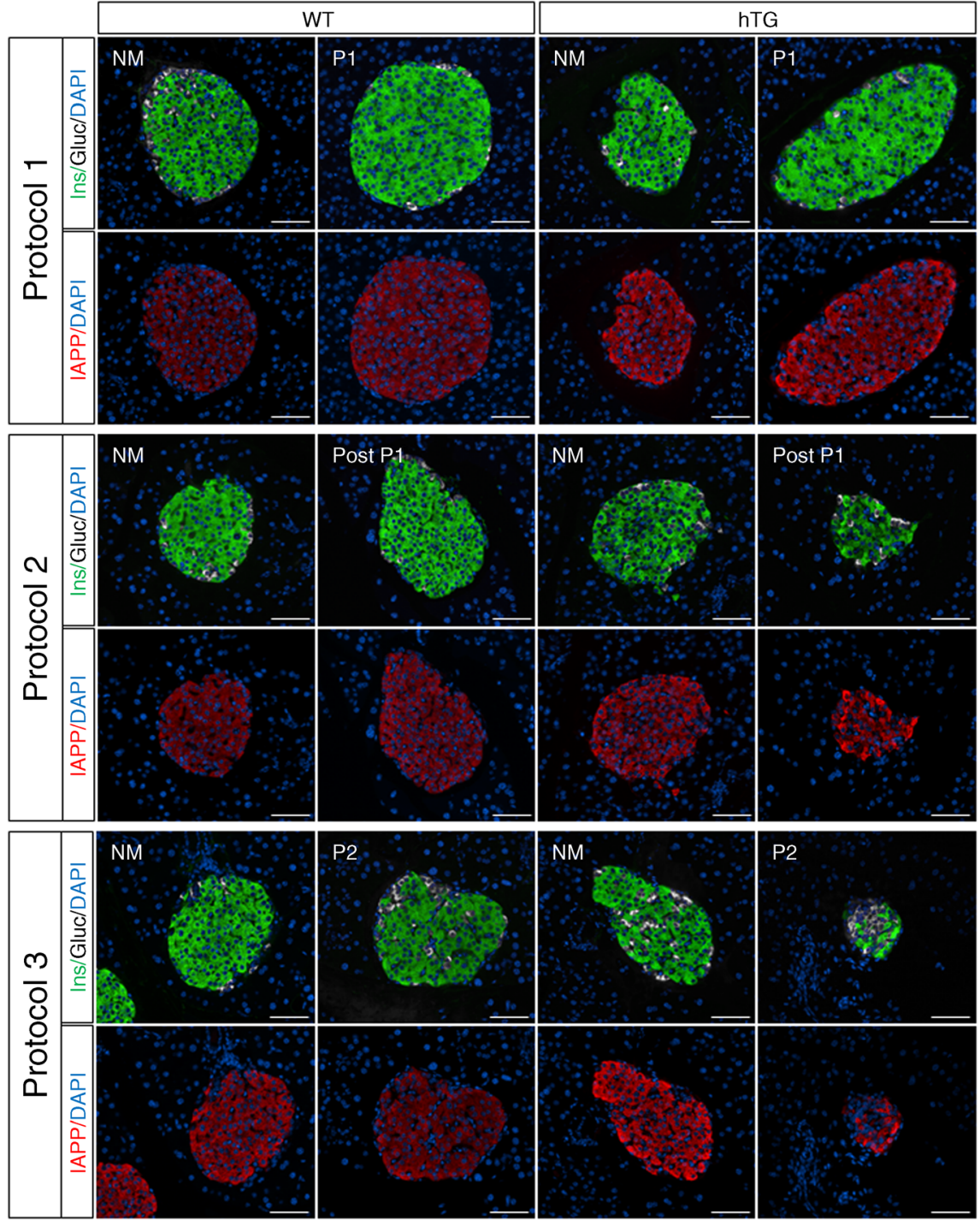

A second pregnancy induces diabetes in hTG mice (Protocol 3) By design, hTG mice included in Protocol 3 were non-diabetic despite a prior pregnancy at 8-11 weeks of age. During the second pregnancy, $\sim 80 \%$ of the hTG mice (7/9) and none of the wild-type mice $(0 / 10)$ developed diabetes. Compared with non-mated control mice, beta cell mass increased $\sim 1.5$-fold in wild-type mice during their second pregnancy $(3.8 \pm 0.5$ vs $2.6 \pm$ $0.3 \mathrm{mg}, p<0.05)$ but decreased by $\sim 70 \%$ in hTG mice during the second pregnancy $(0.8 \pm 0.2$ vs $2.5 \pm 0.3 \mathrm{mg}$, $p<0.01$; Fig. 3b, Protocol 3). The morphology of islets is shown in Fig. 4, Protocol 3. The alpha cell mass was comparable between the groups (ESM Fig. 4).

The mechanism underlying the pregnancy-induced diabetes in hTG mice was an approximately threefold increase in beta cell death compared with the age-matched non-mated control mice $(1.34 \pm 0.24 \%$ vs $0.39 \pm 0.05 \%, p<0.001$; Fig. 3d, Protocol 3).
These data imply that the beta cell toxicity induced by hIAPP misfolding implicated in beta cell failure in type 2 diabetes may contribute to pregnancy-related diabetes. Further, the pregnancy-related hIAPP beta cell stress from sequential pregnancies may be additive. hIAPP misfolding leads to several downstream effects that have been reported in beta cells in type 2 diabetes, including ER stress, accumulated DNA damage and impaired autophagy [29, 30]. Next, we sought to determine whether pregnancy-induced hIAPP toxicity manifests through these effects.

\section{Pregnancy-induced hIAPP beta cell toxicity is characterised by ER stress, impaired autophagy and oxidative stress manifest by accumulated DNA damage}

The marker of ER stress, nuclear immune reactivity to CHOP, was infrequently detected in beta cells in pregnant and non- 
mated wild-type mice (Fig.5). By contrast, nuclear CHOP was readily detectable in non-mated hTG mice and pregnancy increased its frequency $\sim 2.5$-fold $(0.88 \pm 0.19 \%$ vs $0.37 \pm 0.1 \%$, $p<0.01$; Fig. 5 , Protocol 1); in pregnant hTG mice the frequency of CHOP was tenfold that in pregnant wild-type mice $(0.09 \pm 0.03 \%, p<0.001)$. A similar pattern was observed after the first pregnancy (Fig. 5b, Protocol 2) and during the second pregnancy $(1.37 \pm 0.39 \%$ vs $0.48 \pm 0.09 \%$, pregnant hTG mice vs non-mated hTG mice, $p<0.01$; Fig. 5, Protocol 3). Taken together, these data affirm that pregnancy-related beta cell hIAPP toxicity is manifest, at least in part, by increased ER stress.

Beta cell accumulation of p62 immuno-reactive sequestome deposits is a manifestation of impaired flux through the autophagy-lysosome pathway [31]. p62-positive intracellular inclusions were detected in beta cells in both nonmated and mated hTG female mice, but not in wild-type mice (Fig. 6a). The frequency of beta cells with p62 inclusions was $\sim 1.7$-fold higher during the first pregnancy in hTG mice compared with non-mated mice $(7.2 \pm 0.8 \%$ vs $4.2 \pm 0.4 \%$, $p<0.001$; Fig. $6 \mathrm{~b}$, Protocol 1), implying that pregnancy exacerbates the autophagy defect induced by the high expression of hIAPP. During the second pregnancy, the frequency of beta cells containing p62 inclusions increased further in pregnant vs non-mated hTG mice $(21.3 \pm 2.6 \%$ vs $7.3 \pm 1.0 \%$, $p<0.001$; Fig. 6b, Protocol 3).

Accumulated oxidative DNA damage has been reported in beta cells in type 2 diabetes by increased $\mathrm{p}-\mathrm{H} 2 \mathrm{AX}$ nuclear immune reactivity [30]. The frequency of p-H2AX nuclear staining in beta cells (Fig. 6c) was increased in hTG mice compared with wild-type mice in Protocol 1 but its frequency was comparable in non-mated vs pregnant hTG mice $(0.32 \pm$ $0.09 \%$ vs $0.35 \pm 0.04 \%$; Fig. 6d, Protocol 1). However, evidence of beta cell nuclear damage was more abundant in hTG mice in the second pregnancy $(0.57 \pm 0.05 \%$ vs $0.30 \pm 0.05 \%$ $\mathrm{p}-\mathrm{H} 2 \mathrm{AX}$-positive beta cells in pregnant vs non-mated mice, $p<0.001$; Fig. 6d, Protocol 3).

In summary, in mice hIAPP-mediated pregnancy-induced beta cell stress manifests as ER stress, impaired flux through autophagy and increased oxidative DNA damage, comparably to that reported in beta cells of humans with type 2 diabetes.

\section{Discussion}

Gestational diabetes presents risks to both mother and child. While there have been elegant clinical studies of gestational diabetes [3-8], there is a paucity of data at the islet level due to limited access to human pancreas and the lack of a representative animal model of human gestational diabetes.

Since there is a difference in the reported adaptation of beta cell mass to pregnancy in mice vs humans, we investigated whether this is because most studies in young mice are undertaken when the capacity for replication is still high, whereas in humans the capacity for beta cell replication is already diminished by menarche. We resolved this by evaluating the increment in beta cell mass and turnover during pregnancy in young vs aged mice. While the former displayed expanded beta cell mass by twofold during pregnancy, the latter displayed only a 1.3 -fold expansion, a comparable increase
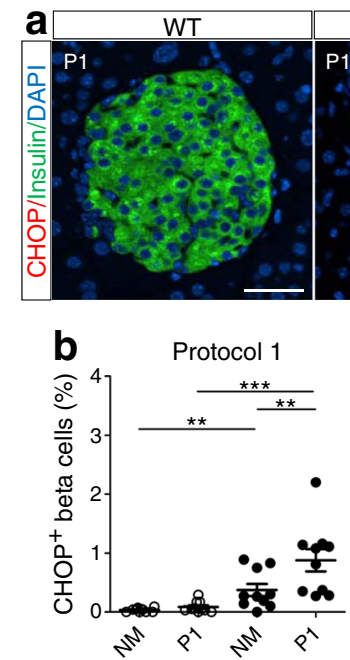
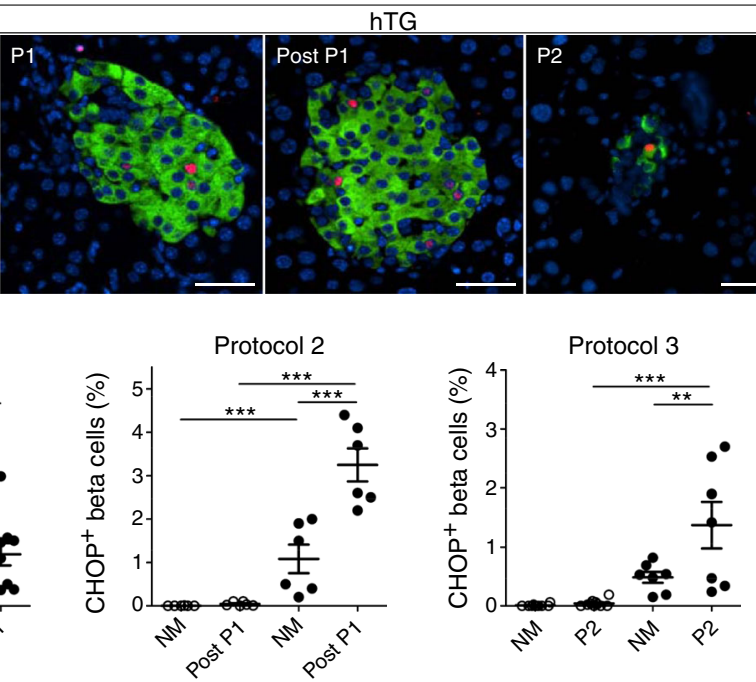

Fig. 5 (a) Representative immunofluorescence images of mouse islets from pancreas samples collected at first pregnancy (P1, Protocol 1), after the first pregnancy (post P1, Protocol 2) and during the second pregnancy (P2, Protocol 3). Sections were stained for insulin (green) and CHOP (red); nuclei were stained blue with DAPI. WT, wild-type. (b) The frequency of beta cells with nuclear CHOP staining in all groups of mice from Protocols 1, 2 and 3. White circles, wild-type mice; black circles, hTG mice. NM, age-matched non-mated mice; P1, first pregnancy; P2, second pregnancy. Data are the mean \pm SEM; $* * p<0.01$ and $* * * p<0.001$, one-way ANOVA followed by Fisher's least-squares difference post hoc test. Protocol 1, $n=10$ for all groups; Protocol 2, $n=6$ for all groups; Protocol 3, $n=7$ in hTG groups and $n=10$ in wild-type groups 

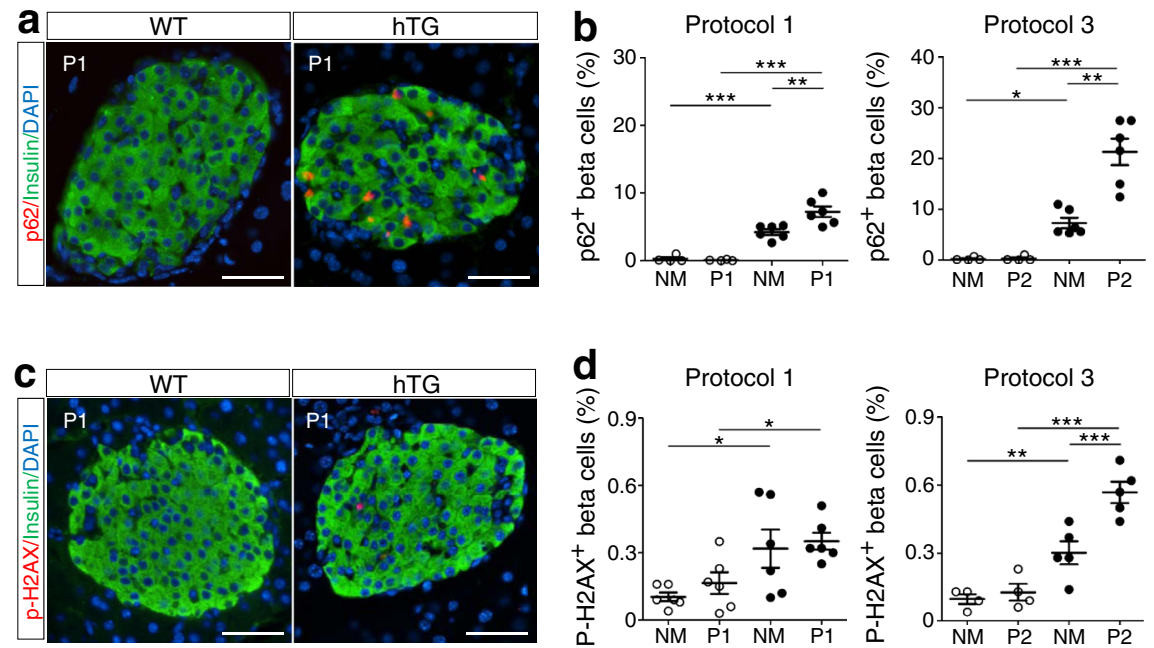

Fig. 6 A defect in autophagy-lysosomal pathway (a, b) and DNA damage $(\mathbf{c}, \mathbf{d})$ are characteristics of beta cells in female hTG mice prone to pregnancy-related diabetes. Representative images of pancreatic sections of wild-type and hTG pregnant and non-mated mice from Protocol 1 (first pregnancy, P1) immunostained for p62 (a) or p-H2AX (c) and insulin were taken using $20 \times$ objective; scale bars, $50 \mu \mathrm{m}$. Quantification of frequency of beta cells positive for the marker of interest $(\mathbf{b}, \mathbf{d})$ is shown for Protocol 1 and Protocol 3 (second pregnancy, P2) and presented as the

to that seen in humans. This difference was due to the agerelated decline in the capacity to increase beta cell replication. The implication is that studies of beta cell adaptation in young pregnant mice are rational if the objective is to study mechanisms regulating beta cell replication, whereas older mice might be more representative of beta cell adaptation in human pregnancy.

Next, we followed up on the observation that female homozygous $\mathrm{h} I A P P$ transgenic breeding mice developed diabetes much more frequently than their non-mated female counterparts. Prior studies have uncovered a protective role of oestrogen against hIAPP-associated beta cell toxicity in this mouse model [20]. Given the lack of murine models of pregnancy-related diabetes, we investigated the impact of a first and second pregnancy on glucose homeostasis, beta cell mass and beta cell turnover in hTG mice. These mice tolerated a first pregnancy without developing diabetes. While no increased beta cell death was recorded at 15 days post coitus during the first pregnancy, this is the peak period of pregnancy-related oestrogen [32] and this might have offset the pregnancy-enhanced hIAPP-mediated ER stress and attenuated autophagy. However, the impact of the pregnancyenhanced hIAPP-mediated beta cell stress became evident in subsequent weeks when the majority of the previously pregnant hTG mice developed diabetes in contrast to none of their non-mated counterparts. This finding implies that a period of transient insulin resistance-induced beta cell misfolded protein stress, such as that induced by pregnancy, can leave residual beta cell vulnerability to subsequent beta cell misfoldinginduced dysfunction and loss. This is consistent with the

mean \pm SEM; $* p<0.05, * * p<0.01$ and $* * * p<0.001$, one-way ANOVA followed by Fisher's least-squares difference post hoc test. White circles, wild-type mice; black circles, hTG mice. NM, age-matched non-mated mice; P1 first pregnancy; P2, second pregnancy; WT, wild-type. In (b) $n=5$ for wild-type groups and $n=6$ for hTG groups; in (d) $n=6$ per group in Protocol 1, $n=4$ for wild-type groups and $n=5$ for hTG groups in Protocol 3

increased risk of type 2 diabetes with multiparity, and the protection against development of type 2 diabetes following gestational diabetes by decreasing the beta cell work load with insulin sensitisers [33].

In the second pregnancy, hTG mice invariably developed diabetes, with markedly greater indices of ER stress, DNA oxidative stress and impaired autophagy leading to increased beta cell apoptosis and loss rather than expansion of beta cell mass. Notably the pregnancy-induced hIAPP-mediated beta cell stress reproduced the ER stress, oxidative damage and impaired autophagy pathways characteristic of beta cells in humans with type 2 diabetes [29, 30]. Moreover, non-mated female hTG counterparts did not develop diabetes.

Efforts to establish rodent models that reproduce gestational diabetes in humans have been problematic [14]. We postulate that this is because rodent IAPP is soluble and does not form toxic oligomers [27]. hIAPP misfolded protein stress is a hallmark of beta cells in type 2 diabetes and, therefore, it is not surprising that the hTG mouse model more closely reproduces human pregnancy-induced diabetes than rodent models that are based on high-fat feeding or leptin deficiency. It should be noted, however, that while it has been established that toxic oligomers of IAPP are present in beta cells in humans with type 2 diabetes, to date there is no data to affirm that this is the case in beta cells in humans with gestational diabetes [19].

Animal models all have limitations in their application to human disease. One obvious difference is the relatively short period of mouse pregnancy with rapid beta cell adaptation compared with the much longer gestational timespan in humans. Some pathways that induce beta cell replication in 
pregnant mice have not been reproduced in humans [34]. While postmenopausal oestrogen replacement has been partially protective against diabetes in humans, there is clearly a much greater protection against beta cell failure and loss afforded by oestrogen in mice. Nonetheless, the hTG mouse model is widely available and reproduces pregnancy-related diabetes in humans to a greater extent than other available rodent models. We propose the hTG mouse as a novel and potentially useful model for investigation of adverse pregnancy-induced changes in beta cells leading to diabetes during and/or subsequent to pregnancy.

Acknowledgements We would like to thank the members of Larry L. Hillblom Islet Research Center at UCLA: B. Lui for editorial assistance, and K. Zeng and M. Cory for technical support. We are thankful to UCLA undergraduate student E. Beebe for help with image analysis.

Data availability The data generated in the studies presented in this publication are available on request without restriction.

Funding The present studies were supported by funding from the United States Public Health Services National Institute of Health grant (DK059579) and the Larry L. Hillblom Foundation (2014-D-001-NET).

Duality of interest The authors declare that there is no duality of interest associated with this manuscript.

Contribution statement TG contributed to the study design, coordinated breeding and tissue collection, performed imaging, image analysis, data analysis and interpretation and wrote the manuscript. SK performed image acquisition and quantitative analysis and contributed to the interpretation of the data. AEB contributed to image analysis and interpretation, critical discussion and reviewing the manuscript. CL contributed to the planning of the study, animal work, tissue collection, tissue staining and image analysis. LP contributed to imaging and image analysis and interpretation of the data. MR contributed to tissue staining, imaging and data analysis. PCB designed the study, interpreted data and wrote the manuscript. All authors contributed to the content and critical revision of the manuscript and agreed to submit the manuscript for publication. TG and PCB are the guarantors of the work.

Publisher's note Springer Nature remains neutral with regard to jurisdictional claims in published maps and institutional affiliations.

\section{References}

1. Catalano PM, Tyzbir ED, Roman NM, Amini SB, Sims EA (1991) Longitudinal changes in insulin release and insulin resistance in nonobese pregnant women. Am J Obstet Gynecol 165:1667-1672

2. Buchanan TA, Metzger BE, Freinkel N, Bergman RN (1990) Insulin sensitivity and $\mathrm{B}$ cell responsiveness to glucose during late pregnancy in lean and moderately obese women with normal glucose tolerance or mild gestational diabetes. Am J Obstet Gynecol 162:1008-1014

3. Buchanan TA (2001) Pancreatic B cell defects in gestational diabetes: implications for the pathogenesis and prevention of type 2 diabetes. J Clin Endocrinol Metab 86:989-993

4. Buchanan TA, Xiang AH (2005) Gestational diabetes mellitus. J Clin Invest 115:485-491
5. Homko C, Sivan E, Chen X, Reece EA, Boden G (2001) Insulin secretion during and after pregnancy in patients with gestational diabetes mellitus. J Clin Endocrinol Metab 86(2):568-573. https:// doi.org/10.1210/jcem.86.2.7137

6. Xiang AH, Peters RK, Trigo E, Kjos SL, Lee WP, Buchanan TA (1999) Multiple metabolic defects during late pregnancy in women at high risk for type 2 diabetes. Diabetes 48:848-854

7. Catalano PM, Huston L, Amini SB, Kalhan SC (1999) Longitudinal changes in glucose metabolism during pregnancy in obese women with normal glucose tolerance and gestational diabetes mellitus. Am J Obstet Gynecol 180:903-916

8. Kautzky-Willer A, Prager R, Waldhausl W et al (1997) Pronounced insulin resistance and inadequate $\beta$-cell secretion characterize lean gestational diabetes during and after pregnancy. Diabetes Care 20: 1717-1723

9. Ben-Haroush A, Yogev Y, Hod M (2004) Epidemiology of gestational diabetes mellitus and its association with type 2 diabetes. Diabet Med 21:103-113

10. Kim C, Newton KM, Knopp RH (2002) Gestational diabetes and the incidence of type 2 diabetes: a systematic review. Diabetes Care 25:1862-1868

11. Naver KV, Lundbye-Christensen S, Gorst-Rasmussen A et al (2011) Parity and risk of diabetes in a Danish nationwide birth cohort. Diabet Med 28:43-47

12. Araneta MR, Barrett-Connor E (2010) Grand multiparity is associated with type 2 diabetes in Filipino American women, independent of visceral fat and adiponectin. Diabetes Care 33:385-389

13. Schwartz N, Green MS, Yefet E, Nachum Z (2016) Modifiable risk factors for gestational diabetes recurrence. Endocrine 54:714-722

14. Plows JF, Yu X, Broadhurst R et al (2017) Absence of a gestational diabetes phenotype in the LepRdb/+ mouse is independent of control strain, diet, misty allele, or parity. Sci Rep 7(1):45130. https:// doi.org/10.1038/srep45130

15. Janson J, Ashley RH, Harrison D, McIntyre S, Butler PC (1999) The mechanism of islet amyloid polypeptide toxicity is membrane disruption by intermediate-sized toxic amyloid particles. Diabetes 48:491-498

16. Matveyenko AV, Gurlo T, Daval M, Butler AE, Butler PC (2009) Successful versus failed adaptation to high-fat diet-induced insulin resistance: the role of IAPP-induced $\beta$-cell endoplasmic reticulum stress. Diabetes 58:906-916

17. Couce M, Kane LA, O'Brien TD et al (1996) Treatment with growth hormone and dexamethasone in mice transgenic for human islet amyloid polypeptide causes islet amyloidosis and $\beta$-cell dysfunction. Diabetes 45:1094-1101

18. Janson J, Soeller WC, Roche PC et al (1996) Spontaneous diabetes mellitus in transgenic mice expressing human islet amyloid polypeptide. Proc Natl Acad Sci U S A 93:7283-7288

19. Gurlo T, Ryazantsev S, Huang CJ et al (2010) Evidence for proteotoxicity in $\beta$ cells in type 2 diabetes: toxic islet amyloid polypeptide oligomers form intracellularly in the secretory pathway. Am J Pathol 176(2):861-869. https://doi.org/10.2353/ajpath. 2010.090532

20. Geisler JG, Zawalich W, Zawalich K et al (2002) Estrogen can prevent or reverse obesity and diabetes in mice expressing human islet amyloid polypeptide. Diabetes 51:2158-2169

21. Karnik SK, Chen H, McLean GW et al (2007) Menin controls growth of pancreatic $\beta$-cells in pregnant mice and promotes gestational diabetes mellitus. Science 318:806-809

22. Butler AE, Cao-Minh L, Galasso R et al (2010) Adaptive changes in pancreatic beta cell fractional area and beta cell turnover in human pregnancy. Diabetologia 53:2167-2176

23. Van Assche FA, Aerts L, De Prins F (1978) A morphological study of the endocrine pancreas in human pregnancy. Br J Obstet Gynaecol 85(11):818-820. https://doi.org/10.1111/j.1471-0528. 1978.tb15835.x 
24. Meier JJ, Butler AE, Saisho Y et al (2008) $\beta$-cell replication is the primary mechanism subserving the postnatal expansion of $\beta$-cell mass in humans. Diabetes 57:1584-1594

25. Tschen SI, Dhawan S, Gurlo T, Bhushan A (2009) Age-dependent decline in $\beta$-cell proliferation restricts the capacity of beta-cell regeneration in mice. Diabetes 58:1312-1320

26. Teta M, Long SY, Wartschow LM, Rankin MM, Kushner JA (2005) Very slow turnover of $\beta$-cells in aged adult mice. Diabetes 54: 2557-2567

27. Huang CJ, Haataja L, Gurlo T et al (2007) Induction of endoplasmic reticulum stress-induced $\beta$-cell apoptosis and accumulation of polyubiquitinated proteins by human islet amyloid polypeptide. Am J Physiol Endocrinol Metab 293:E1656-E1662

28. Butler AE, Janson J, Bonner-Weir S, Ritzel R, Rizza RA, Butler PC (2003) Beta-cell deficit and increased $\beta$-cell apoptosis in humans with type 2 diabetes. Diabetes 52:102-110

29. Huang CJ, Lin CY, Haataja L et al (2007) High expression rates of human islet amyloid polypeptide induce endoplasmic reticulum stress mediated $\beta$-cell apoptosis, a characteristic of humans with type 2 but not type 1 diabetes. Diabetes 56:2016-2027
30. Mizukami H, Takahashi K, Inaba W et al (2014) Involvement of oxidative stress-induced DNA damage, endoplasmic reticulum stress, and autophagy deficits in the decline of $\beta$-cell mass in Japanese type 2 diabetic patients. Diabetes Care 37:1966-1974

31. Rivera JF, Costes S, Gurlo T, Glabe CG, Butler PC (2014) Autophagy defends pancreatic $\beta$-cells from human islet amyloid polypeptide-induced toxicity. J Clin Invest 124:3489-3500

32. Barkley MS, Geschwind II, Bradford GE (1979) The gestational pattern of estradiol, testosterone and progesterone secretion in selected strains of mice. Biol Reprod 20:733-738

33. Buchanan TA, Xiang AH, Peters RK et al (2002) Preservation of pancreatic $\beta$-cell function and prevention of type 2 diabetes by pharmacological treatment of insulin resistance in high-risk hispanic women. Diabetes 51:2796-2803

34. Baeyens L, Hindi S, Sorenson RL, German MS (2016) $\beta$-Cell adaptation in pregnancy. Diabetes Obes Metab 18(Suppl 1):6370. https://doi.org/10.1111/dom.12716 